\title{
Modelo De Sistema Viable - Modelo De Articulación Del Sistema De Gestión Integral Hseq Bajo Los Criterios De Las Normas Iso 9001:2015, Iso 14001: 2015 E Iso 45001:2018
}

Calderón Pino Anggie Daniela dol , López Mendoza Miguel Ángel, Romero Hernández María Alejandra.

Universidad Autónoma de Colombia, Bogotá, Colombia

Recibido: 08/10/2020 Revisado: 15/10/2020Ａceptado: 15/10/2020 Publicado: 28/01/2021

\section{RESUMEN}

El modelo de sistema viable es un modelo que busca ser implementado dentro de todas las organizaciones independientemente de su actividad; este busca generar autonomía en todas las partes de la organización a través de un modelo replicado de integración sinergia y cohesión.

Este modelo se conforma por Mecanismos de cohesión en donde encontramos el canal de comandos, coordinación y el monitoreo de las actividades primarias; y el mecanismo de adaptación donde encontramos las políticas, inteligencia el control y cohesión.

Este modelo de sistema viable permite una correcta comunicación entre todas las áreas de la empresa a través de interacciones entre actividades todas apuntando al mismo objetivo.

Lo que se pretende realizar es implementar una lista de verificación que nos sirva como diagnostico dentro de las organizaciones e identifique las principales áreas en donde se está cumpliendo a cabalidad el sistema de modelo viable y cuales son aquellas donde presenta deficiencias.

Lo que se pretende con estos hallazgos es asegurar la integración entre todas las partes involucradas; creando sinergia y eficiencia mediante un mismo sistema viable conectado y replicado en cada área.

\section{PALABRAS ClAVES}

Identidad, Cohesión, adaptación, desempeño, ciudadanía. 


\begin{abstract}
The viable system model is a model that seeks to be implemented within all organizations regardless of their activity; This seeks to generate autonomy in all parts of the organization through a replicated model of integration, synergy and cohesion.

This model is made up of Cohesion Mechanisms where we find the command channel, coordination and monitoring of primary activities; and the adaptation mechanism where we find policies, intelligence, control and cohesion.
\end{abstract}

This viable system model allows correct communication between all areas of the company through interactions between activities, all aiming at the same objective.

What is intended to be done is to implement a checklist that serves as a diagnosis within the organizations and identifies the main areas where the viable model system is being fully complied with and which are those where it has deficiencies.

What is intended with these findings is to ensure integration between all parties involved; creating synergy and efficiency through the same viable system connected and replicated in each area.

\title{
KEYWORDS
}

Identity, Cohesion, adaptation, performance, citizenship. 


\section{INTRODUCCIÓN}

En el siguiente informe se presenta el Modelo de sistema viable (MSV) como un modelo de diseño y diagnostico organizacional, el cual pretende mejorar el funcionamiento de las empresas a través de la integración y sinergia de los mecanismos de cohesión y adaptación replicados en todas las áreas de la empresa.

Igualmente permite conocer e interpretar los mecanismos de flexibilidad y adaptabilidad de las organizaciones, permitiendo articular sus objetivos y relaciones entre los actores relevantes; promoviendo el crecimiento y permanecía de una organización en el mercado a través del tiempo. Permite identificar las áreas donde se requiere tomar acciones de mejora

\section{ASPECTOS TEÓRICOS}

\section{¿QUE ES EL MODELO DE SISTEMA VIABLE?}

Modelo con la capacidad de detectar los cambios del entorno adaptándose a las condiciones inesperadas de forma rápida y oportuna permitiéndole sobrevivir a través del tiempo gracias a su flexibilidad y adaptabilidad.

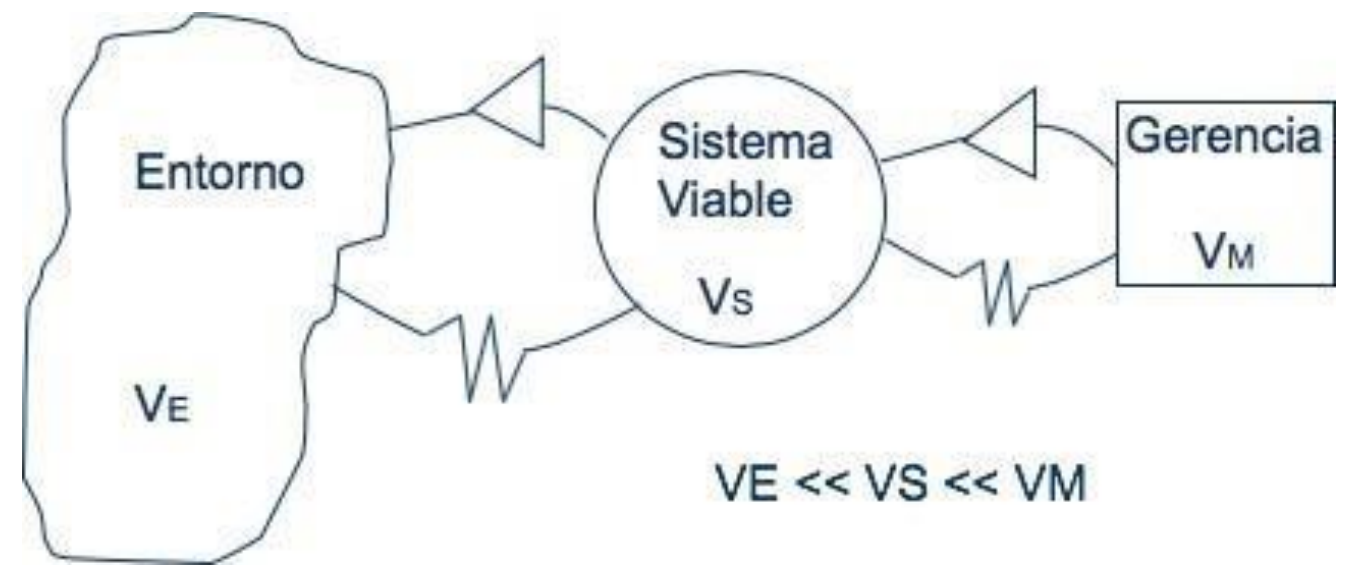

Figura 1. Gerencia de complejidad (Espejo, R. 2003, Pérez, G. 2020)

\section{ENTIDADES FUNDAMENTALES:}


EL ENTORNO "es todo lo que es externo al sistema y le es relevante. Para una empresa, el entorno es el sector económico en el que se encuentra, los factores sociales que la condicionan, las circunstancias económicas y políticas que la rodean, etc.” (Beer S. , 1985)

LA OPERACIÓN "representa todas las actividades que producen el sistema y le dan su significado. Cada una de estas operaciones puede constituir un sistema viable en sí misma (de ahí la recursividad del modelo)"

LA GESTIÓN: "representa todas las actividades de dirección necesarias para hacer funcionar el sistema. A diferencia de lo que sucede con las operaciones, la gestión no se puede considerar como un sistema viable, pues no tiene capacidad de existencia en sí misma”. (Beer S. , 1985) 


\section{GERENCIA DE COMPLEJIDAD}

\section{Mecanismo de Cohesión:}

- Canal de Comandos

- Canal de Coordinación

- Actividades primarias operaciones

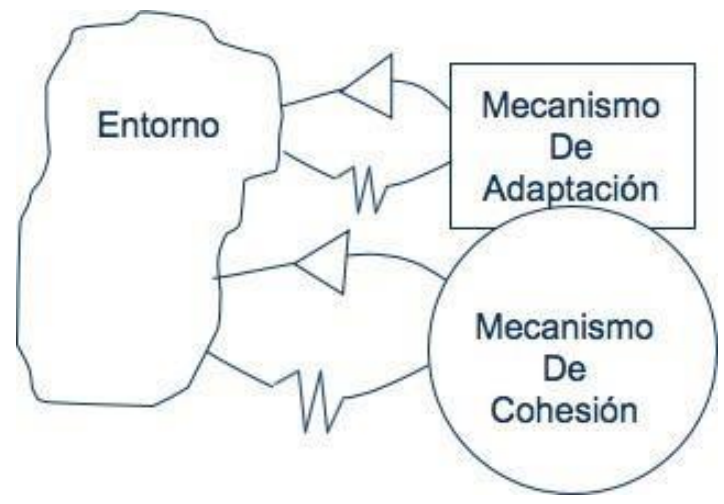

Figura 2. Gerencia de complejidad (Espejo, R. 2003, Pérez, G. 2020)

\section{Mecanismos de Adaptación:}

- Políticas

- Inteligencia

- Control y cohesión

\section{CANAL DE COMANDOS.}

Determina lo que hace el sistema

$\checkmark$ Identificar la organización y dar nombre.

$\checkmark$ Determinar la actividad que desarrolla

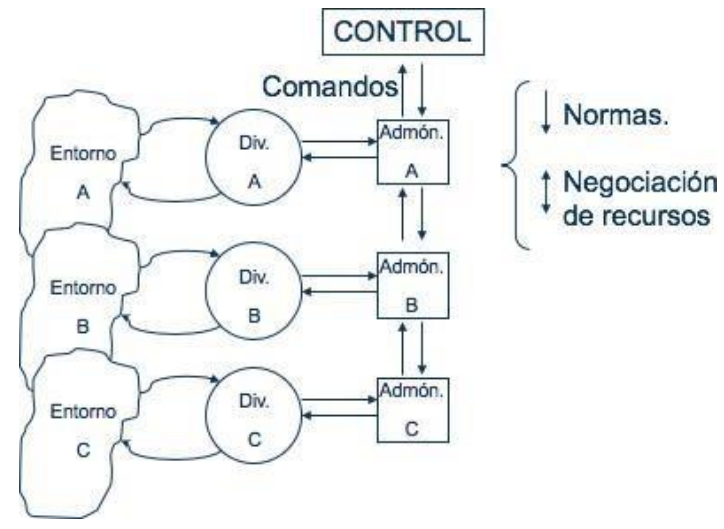
el sistema 


\section{CANAL DE COORDINACIÓN}

*Es la encargada de minimizar las descoordinaciones entre las áreas.

*Crea acuerdo entre los subsistemas para beneficio del fin.

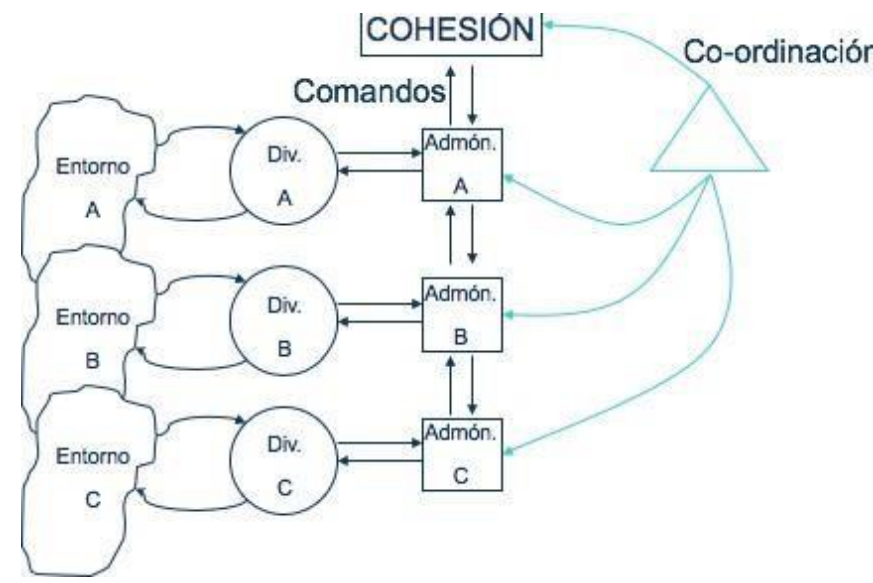

Figura 4. Mecanismo de Cohesión. Canal de Coordinación.

(Espejo, R. 2003, Pérez, G. 2020)

su función es garantizar que todos los subsistemas tengan el mismo lenguaje y trabajen por el mismo objetivo.

\section{ACTIVIDADES PRIMARIAS.}

> Actividades Tecnológicas: Actividades destinadas a construir los productos o servicios que constituyen la razón de ser de la organización

Actividades Reguladoras: Actividades de administración y apoyo a las actividades anteriores". (El Modelo de Sistema Viable, s.f.)

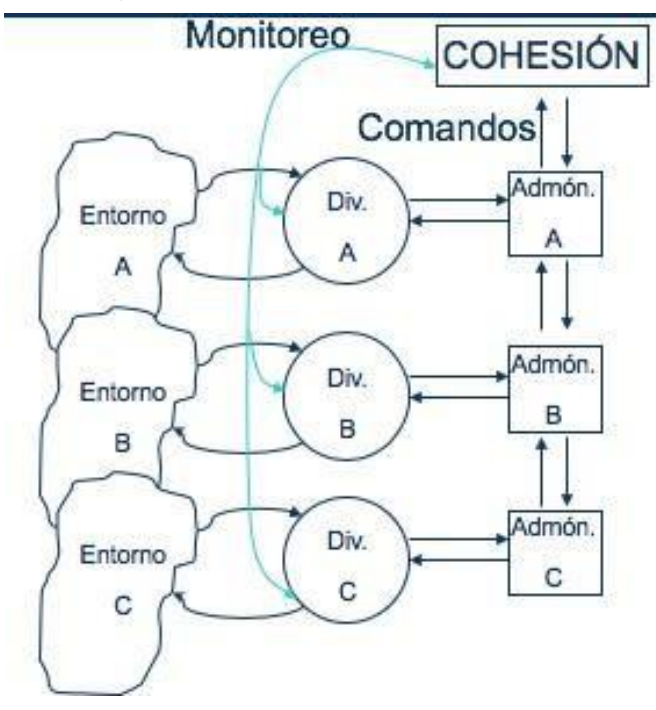

Figura 5. Mecanismo de Cohesión. Monitoreo de Actividades primarias. (Espejo, R. 2003, Pérez, G. 


\section{MECANISMO DE COHESIÓN COMPLETO}

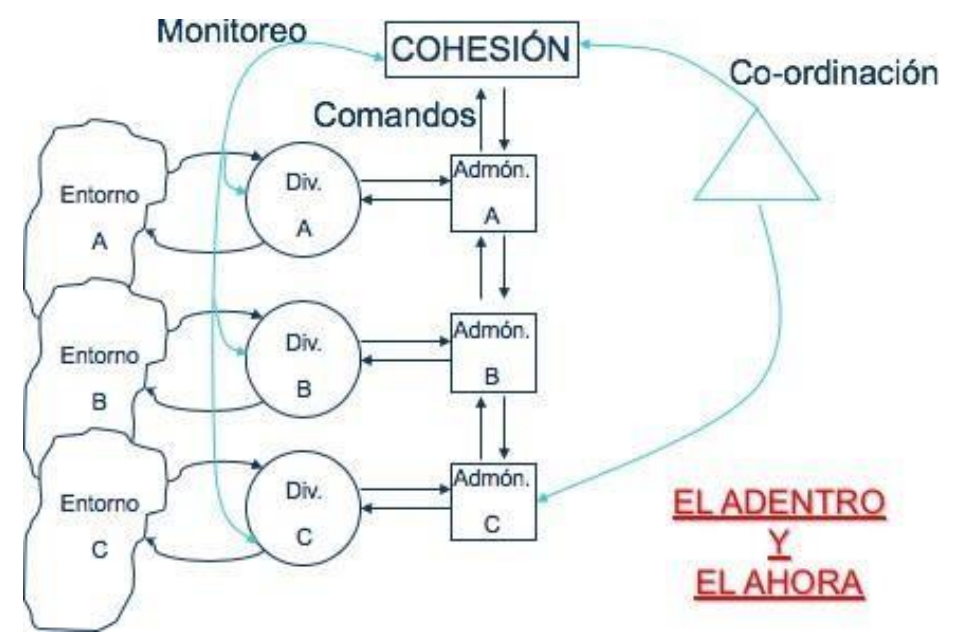

$\checkmark$ Minimizar el uso del canal de comandos.

$\checkmark$ Usar el canal de monitores de forma esporádica y con discreción

$\checkmark$ Maximizar la coordinación entre actividades primarias.

Figura 6. Mecanismo de Cohesión. Completo. (Espejo, R. 2003, Pérez, G. 2020)

\section{MECANISMO DE ADAPTACIÓN COMPLETO.}

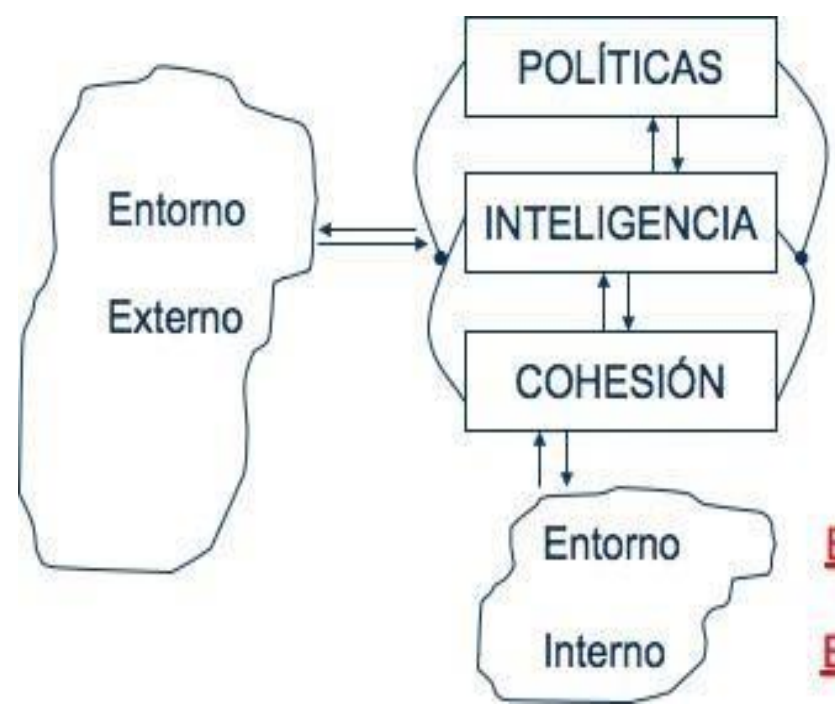

$\checkmark$ Minimizar los requerimientos de información de quienes definen las políticas.

$\checkmark$ Diseñar las funciones de inteligencia y cohesión con una complejidad similar.

$\checkmark$ Asegurar una alta $\frac{\text { ELAFUERA interrelación entre las }}{\mathrm{Y}}$ ELFUTURO funciones de inteligencia $y$ cohesión.

Figura 7. Mecanismo de Adaptación. Completo. (Espejo, R. 2003, Pérez, G. 202 


\section{POLÍTICA, INTELIGENCIA Y CONTROL:}

La Política está directamente relacionada con la alta dirección y la ejecución de las actividades primarias; la política debe tener la capacitar de dar soluciones a situaciones de riesgo cotidianas; de tal forma que la organización pueda adaptarse rápidamente a los cambios del entorno.

La inteligencia debe ir directamente ligada con el entorno y estar en constante actualización y desarrollo de las nuevas tecnologías de acuerdo a las nuevas necesidades que surgen con el paso del tiempo.

\section{MSV VIABLE COMPLETO}

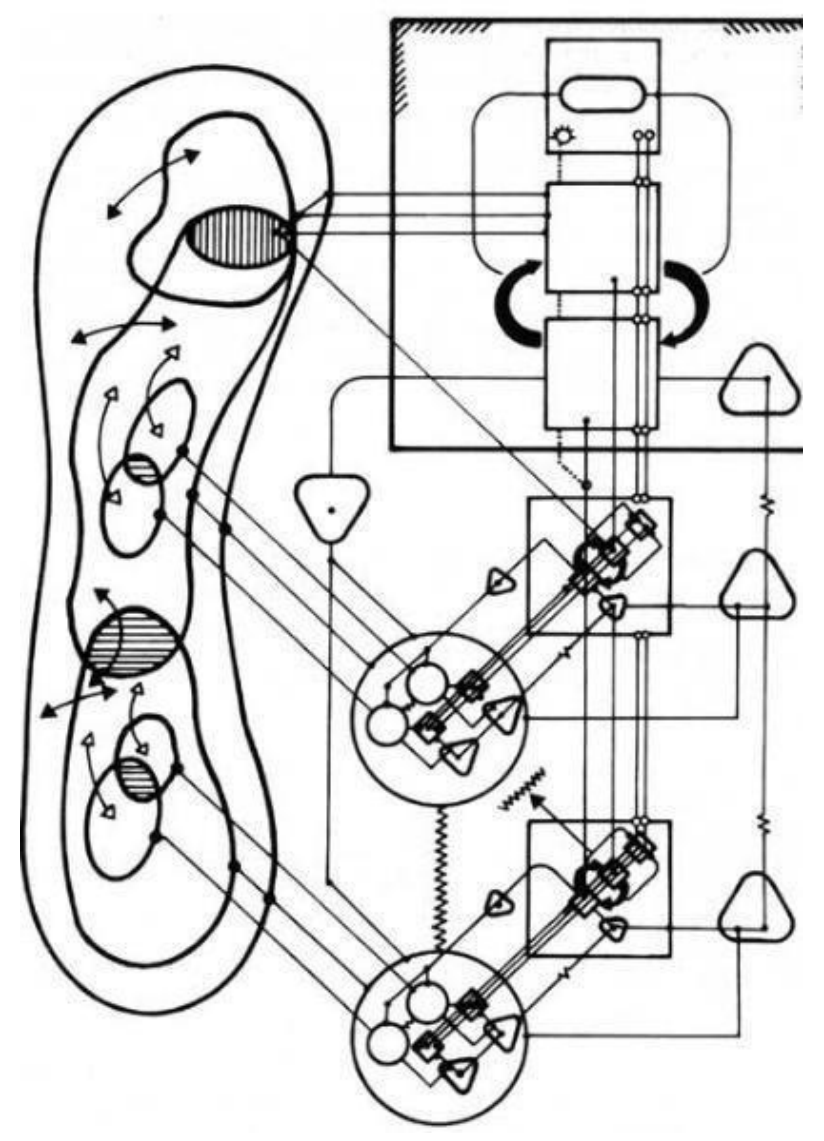

Figura 8. El modelo del Sistema Viable al completo. (Beer, S. 1985) 


\section{DESARROLLO}

"El MSV permite abordar la enorme complejidad (variedad) a la que se enfrentan las organizaciones" (Beer S. , 1979). Estas son asociadas a la implementación, Coordinación, integración, inteligencia y política; con estas se pretende lograr un MSV dentro de una organización.

Con el objeto de lograr identificar el porcentaje de viabilidad en la que se encuentra una organización se desarrolla una herramienta de verificación que permite medir el nivel de viabilidad de cada uno de los comandos que se proponen dentro de un MSV (Mecanismos de cohesión en donde encontramos el canal de comandos, coordinación y el monitoreo de las actividades primarias; y el mecanismo de adaptación donde encontramos las políticas, inteligencia el control y cohesión.).

Esta lista de verificación les permitirá a todas las organizaciones detectar cuáles son las áreas donde presenta un mayor porcentaje de viabilidad y cuales son aquellas donde se requieren tomar decisiones y realizar cambios que permitan alinearse al MSV al que se pretende llegar dentro de la organización.

La lista de verificación está planeada de acuerdo a los comandos identificados por Stafford Beer y "permite examinar aspectos estructurales del programa por cuadrantes como un lenguaje unificador de la sostenibilidad en organizaciones de diferentes tipos y escalas". (Espinosa \& Walker, A complexity approach to sustainability: Theory and application, 2011) 
Los criterios de calificación que se va a utilizar serán los siguientes:

\begin{tabular}{|c|c|c|c|c|c|}
\hline \multicolumn{6}{|c|}{ CRITERIOS DE CALIFICACION } \\
\hline \multirow{2}{*}{ ESCALA } & \multirow{2}{*}{ ABREV. } & \multirow{2}{*}{ DESCRIPCIÓN } & \multicolumn{3}{|c|}{ INTERVALO } \\
\hline & & & Debil & Medio & Alto \\
\hline ASIMILADO Y MEJORADO & M & La tarea además, se mejora & 10 & 11 & 12 \\
\hline ANALIZADO Y COMPRENDIDO & A & La tarea se ejecuta y se controla & 7 & 8 & 9 \\
\hline IMPLANTADO & I & Está implementada la tarea & 4 & 5 & 6 \\
\hline SÓLIDAMENTE FUNDAMENTADO & S & Está definida y documentada la tarea & 1 & 2 & 3 \\
\hline NO EXISTE. NO APLICA & $\mathrm{N}$ & No está definida la tarea & \multicolumn{3}{|c|}{0} \\
\hline
\end{tabular}

Tabla 1: Criterios de calificación (perez Gomez,

Con estos criterios de calificación se pretende dar a conocer el porcentaje de viabilidad en el que se encuentra el MSV el cual contiene un "conjunto de partes interrelacionadas que percibimos Como un todo" (Espejo \& Reyes, 2016, pág. 16)

"La metodología se enfoca en la capacidad de resiliencia de los sistemas, es decir, en la superación desde adentro del sistema de los problemas" (Espinosa \& Walker, A complexity approach to sustainability: Theory and application, 2011). Logrando que sea viable; "asegurando sus capacidades de existencia independiente, autorregulación, aprendizaje, adaptación y evolución necesarias para garantizar su supervivencia” (Puche Regaliza, Pérez Ríos, \& Sánchez Mayoral, 2005).

La lista de verificación se diligenciará con una escala de 0 a 12 en donde 0 No existe y 12 esta asimilado y mejorado; lo que se pretende es que las organizaciones contesten las preguntas utilizando de forma correcta la escala planteada en la Tabla 1 y lo realicen teniendo como soporte la información documentada de la organización. 
A continuación, se detallan las listas de preguntas que se desarrollaron para ser aplicadas dentro de las organizaciones; estas están clasificadas de acuerdo a cada Mecanismo:

\section{MECANISMOS DE COHESIÓN}

\section{CANAL DE COMANDOS}

\begin{tabular}{|l|l|}
\hline $\mathbf{1}$ & $\begin{array}{l}\text { ¿Qué tanto conoce usted de las funciones y/o actividades } \\
\text { que desarrolla? }\end{array}$ \\
\hline $\mathbf{2}$ & $\begin{array}{l}\text { ¿En qué nivel las actividades que desarrolla contienen } \\
\text { documentación que garantice la ejecución de sus } \\
\text { funciones diarias con calidad? }\end{array}$ \\
\hline $\mathbf{3}$ & $\begin{array}{l}\text { ¿Cuán útil considera que son necesarios los recursos } \\
\text { como manuales e instructivos, en el desarrollo de su } \\
\text { actividad, para un correcto desempeño? }\end{array}$ \\
\hline $\mathbf{4}$ & $\begin{array}{l}\text { ¿En qué nivel le ayudan los procedimientos y procesos } \\
\text { instructivos para desarrollar su trabajo? }\end{array}$ \\
\hline $\mathbf{5}$ & $\begin{array}{l}\text { ¿Bajo qué nivel las inducciones y/o capacitaciones le } \\
\text { permiten tener claridad del adecuado procedimiento que } \\
\text { se debe llevar a cabo en la realización de los procesos de } \\
\text { la empresa? }\end{array}$ \\
\hline $\mathbf{6}$ & $\begin{array}{l}\text { ¿Qué tanto conoce de la existencia del manual de procesos } \\
\text { que desarrolla la empresa? } \\
\text { ¿De conocer el manual de procesos de la empresa, en qué }\end{array}$ \\
\hline
\end{tabular}

COORDINACIÓN: "Es el encargado de sincronizar las diferencias que se puedan Presentar en cada uno de los subsistemas, garantizando que todos tengan un lenguaje Común con objetivos institucionales y no locales". (Espinosa \& Walker, A complexity approach to sustainability: Theory and application, 2011) 


\begin{tabular}{|l|l|}
\hline $\mathbf{1}$ & $\begin{array}{l}\text { ¿Considera que la programación de trabajo es acorde a lo } \\
\text { planeado por los superiores? }\end{array}$ \\
\hline $\mathbf{2}$ & $\begin{array}{l}\text { ¿En qué escala considera usted que el plan de trabajo está } \\
\text { enfocado a los objetivos solicitados? }\end{array}$ \\
\hline $\mathbf{3}$ & $\begin{array}{l}\text { ¿Qué tan claro tiene usted las funciones que debe } \\
\text { desarrollar en su puesto de trabajo? }\end{array}$ \\
\hline $\mathbf{4}$ & $\begin{array}{l}\text { ¿En qué escala considera usted que conoce como se } \\
\text { realiza la planeación y programación de las operaciones? }\end{array}$ \\
\hline $\mathbf{5}$ & $\begin{array}{l}\text { ¿Qué tanto considera usted que sus funciones en el proceso } \\
\text { de producción tienen impacto para la funcionalidad de la } \\
\text { operación? }\end{array}$ \\
\hline $\mathbf{6}$ & $\begin{array}{l}\text { ¿Considera usted que las tareas que elabora están acordes } \\
\text { con el planeamiento de la producción? }\end{array}$ \\
\hline $\mathbf{7}$ & $\begin{array}{l}\text { ¿Los mecanismos de control de los procesos productivos } \\
\text { son los ideales, en que escala los ubica? }\end{array}$ \\
\hline $\mathbf{8}$ & $\begin{array}{l}\text { ¿Qué tan claro tiene usted el rol que cumple en el proceso } \\
\text { productivo? }\end{array}$ \\
\hline
\end{tabular}

ACTIVIDADES PRIMARIAS: "Siendo estas unidades funcionales las encargadas de desarrollar las áreas y actividades básicas de la organización”. (Peón Escalante, 2015)

\begin{tabular}{|l|l|}
\hline $\mathbf{1}$ & $\begin{array}{l}\text { ¿Las actividades se ejecutan de acuerdo con lo establecido } \\
\text { en los procedimientos e instructivos? }\end{array}$ \\
\hline $\mathbf{2}$ & $\begin{array}{l}\text { ¿La organización cuenta con los procedimientos } \\
\text { adecuados en cada actividad garantizando la satisfacción } \\
\text { de los requerimientos del cliente? }\end{array}$ \\
\hline $\mathbf{3}$ & $\begin{array}{l}\text { ¿Las operaciones se realizan cumpliendo con los } \\
\text { requerimientos y tiempos establecidos en los procesos y } \\
\text { procedimientos? }\end{array}$ \\
\hline $\mathbf{4}$ & $\begin{array}{l}\text { ¿Se realiza medición y seguimiento a las actividades } \\
\text { asegurando el resultado final? }\end{array}$ \\
\hline
\end{tabular}




\begin{tabular}{|c|c|}
\hline 5 & $\begin{array}{l}\text { El seguimiento de las actividades se verifica de forma } \\
\text { bjetiva garantizando la imparcialidad y el cumplimiento } \\
\text { e los procedimientos? }\end{array}$ \\
\hline 6 & $\begin{array}{l}\text { ¿Existen, Se conocen y cumplen los procedimientos de } \\
\text { contingencias que garanticen la continuidad del negocio } \\
\text { en situaciones de riesgo? }\end{array}$ \\
\hline 7 & $\begin{array}{l}\text { ¿Cuándo se detectan riesgos en las actividades } \\
\text { desarrolladas dentro de la organización se toman las } \\
\text { acciones correctivas documentadas en los procedimientos } \\
\text { de contingencias? }\end{array}$ \\
\hline 8 & $\begin{array}{l}\text { ¿El desarrollo y resultado de las actividades se conserva } \\
\text { documentado como evidencia de los resultados? }\end{array}$ \\
\hline 9 & $\begin{array}{l}\text { ¿Existe disponibilidad, confiabilidad e integridad en la } \\
\text { información financiera y operativa de la organización? }\end{array}$ \\
\hline 10 & $\begin{array}{l}\text { ¿La organización lleva a cabo auditorías internas } \\
\text { conforme a los procedimientos e instructivos establecidos } \\
\text { para cada actividad? }\end{array}$ \\
\hline 11 & $\begin{array}{l}\text { La organización planifica, establece, ¿implementa y } \\
\text { mantiene uno o varios programas de auditoría? }\end{array}$ \\
\hline 12 & $\begin{array}{l}\text { ¿Los resultados de las auditorias son documentados e } \\
\text { informados a la alta dirección? }\end{array}$ \\
\hline
\end{tabular}

\section{MECANISMOS DE ADAPTACIÓN}

5.2.1. POLÍTICAS: "Es el proceso que regula la estabilidad interna frente al cambio externo, siendo el que define el ritmo de cambio adecuado del sistema, al regular la relación del sistema con su medio. Es decir, es un proceso de sabiduría regulador del ritmo de cambio”. (Peón Escalante, 2015) 


\begin{tabular}{|l|l|}
\hline $\mathbf{1}$ & $\begin{array}{l}\text { ¿La organización logra identificar las señales del entorno } \\
\text { y realiza los ajustes necesarios para adaptarse a las nuevas } \\
\text { exigencias del entorno? }\end{array}$ \\
\hline $\mathbf{2}$ & $\begin{array}{l}\text { ¿La alta dirección establece el horizonte (metas a seguir) } \\
\text { de acuerdo con las variaciones que ocurren en el día a día? }\end{array}$ \\
\hline $\mathbf{3}$ & $\begin{array}{l}\text { ¿La alta dirección realiza programaciones frecuentes para } \\
\text { revisar los resultados esperados en cada directriz y realizar } \\
\text { los respectivos ajustes? (Indique en observaciones los } \\
\text { horarios cotidianos que manejan). }\end{array}$ \\
\hline $\mathbf{4}$ & $\begin{array}{l}\text { ¿La alta dirección realiza acciones para formular nuevas } \\
\text { directrices de trabajos en donde se estructuran las nuevas } \\
\text { estrategias de aplicación cotidiana? }\end{array}$ \\
\hline $\mathbf{5}$ & $\begin{array}{l}\text { ¿La alta dirección tiene pleno conocimiento de la } \\
\text { ejecución, seguimiento y medición de las acciones } \\
\text { indicadas para mantener la operación en el curso } \\
\text { esperado? }\end{array}$ \\
\hline $\mathbf{6}$ & $\begin{array}{l}\text { ¿De los comités que se realizan surgen las alternativas de } \\
\text { solución a los problemas que se presentan diariamente? }\end{array}$ \\
\hline $\mathbf{7}$ & $\begin{array}{l}\text { ¿La alta dirección tiene la capacidad de impartir } \\
\text { directrices apropiadas a ejecutar cuando se presentan } \\
\text { situaciones de perturbación en las actividades cotidianas? }\end{array}$ \\
\hline $\mathbf{8}$ & $\begin{array}{l}\text { Cuando se detecta un problema inesperado el cual requiere } \\
\text { de atención inmediata; ila alta dirección toma las } \\
\text { directrices adecuadas de forma rápida y oportuna? }\end{array}$ \\
\hline $\mathbf{9}$ & $\begin{array}{l}\text { ¿La alta dirección establece políticas de rentabilidad y } \\
\text { competitividad alineadas a los nuevos desafíos del } \\
\text { entorno? }\end{array}$ \\
\hline $\mathbf{1 0}$ & $\begin{array}{l}\text { ¿La alta dirección imparte directrices que permiten tener } \\
\text { el impacto ambiental controlado? }\end{array}$ \\
\hline
\end{tabular}




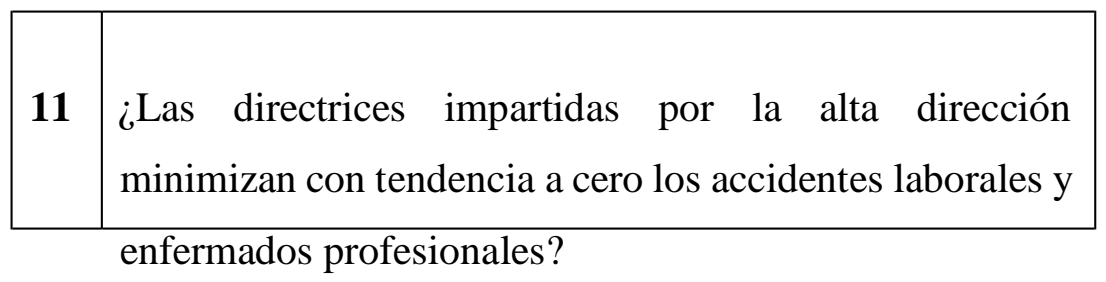

INTELIGENCIA: "Es responsable del "afuera y luego". Su enfoque es el mundo exterior, va en busca de amenazas y oportunidades y viene con planes para adaptarse a las nuevas circunstancias”. (Espinosa \& Walker, 2017)

\begin{tabular}{|l|l|}
\hline $\mathbf{1}$ & $\begin{array}{l}\text { ¿Qué tanto considera que el equipo de innovación ciencia } \\
\text { y tecnología se enfoca en las tendencias y vanguardias } \\
\text { actuales? }\end{array}$ \\
\hline $\mathbf{2}$ & $\begin{array}{l}\text { ¿Qué tan eficiente considera que el sistema (software) } \\
\text { manejado para los procesos de la empresa es? }\end{array}$ \\
\hline $\mathbf{3}$ & $\begin{array}{l}\text { ¿La empresa logra adaptarse rápidamente a los cambios } \\
\text { de su ambiente externo e interno y busca un cambio para } \\
\text { satisfacer este requerimiento? En qué escala la ubica }\end{array}$ \\
\hline $\mathbf{4}$ & $\begin{array}{l}\text { ¿Qué tanto considera que la empresa se encuentra } \\
\text { innovando? }\end{array}$ \\
\hline $\mathbf{5}$ & $\begin{array}{l}\text { ¿En qué escala considera usted que la cultura de la } \\
\text { empresa invita a la innovación? }\end{array}$ \\
\hline $\mathbf{6}$ & $\begin{array}{l}\text { ¿Las capacidades de la empresa son las aptas para estar en } \\
\text { constante innovación? En qué escala la ubica }\end{array}$ \\
\hline $\mathbf{7}$ & $\begin{array}{l}\text { ¿El trabajo de investigación externa y adaptación de la } \\
\text { compañía en que escala considera que esta? }\end{array}$ \\
\hline $\mathbf{8}$ & $\begin{array}{l}\text { ¿Considera que la modernización y actualización de los } \\
\text { sistemas de información y plataformas son los adecuados? }\end{array}$ \\
\hline
\end{tabular}

CONTROL Y COHESIÓN: Representa las estructuras y controles que se ponen en marcha para establecer las reglas, recursos, derechos y responsabilidades del sistema de las actividades primaria y proporcionar una interfaz con los sistemas coordinación, inteligencia y políticas". (Francis, Francis, Taylor, \& Kinloch, 2009). 


\begin{tabular}{|l|l|}
\hline $\mathbf{1}$ & $\begin{array}{l}\text { ¿En qué nivel usted, mediante su rol, colabora para que } \\
\text { el sistema opere de forma óptima? }\end{array}$ \\
\hline $\mathbf{2}$ & $\begin{array}{l}\text { ¿Qué control se realiza a los productos y/o servicios } \\
\text { ofrecidos por la empresa que permita eliminar las no } \\
\text { conformidades? }\end{array}$ \\
\hline $\mathbf{3}$ & $\begin{array}{l}\text { ¿En qué nivel se produce calidad y seguridad en los } \\
\text { productos y/o servicios ofrecidos por la empresa que den } \\
\text { cumplimiento a la normatividad de cada país? }\end{array}$ \\
\hline $\mathbf{4}$ & $\begin{array}{l}\text { ¿Con que frecuencia se generan auditorias periódicas en } \\
\text { cada área de la empresa, que permitan identificar } \\
\text { inconsistencias y generar mejoras? }\end{array}$ \\
\hline $\mathbf{5}$ & $\begin{array}{l}\text { ¿Qué nivel de trazabilidad en los procesos se ofrece en los } \\
\text { productos y/o servicios entregados? }\end{array}$ \\
\hline $\mathbf{6}$ & $\begin{array}{l}\text { ¿Con que frecuencia realiza seguimiento y corrección de } \\
\text { inconsistencias detectadas en las auditorias? }\end{array}$ \\
\hline $\mathbf{7}$ & $\begin{array}{l}\text { ¿Qué factibilidad ejerce el plan estratégico de control de } \\
\text { las operaciones en la empresa? }\end{array}$ \\
\hline
\end{tabular}

Las preguntas anteriores representan base de la lista de verificación desarrollada; la cual será utilizada como herramienta diagnóstico dentro de las organizaciones; con el fin de determinar el porcentaje de viabilidad de la organización e identificar los comandos que requieren una intervención inmediata, para lograr alinearse al MSV esperado dentro de la organización.

Si se logra alcanzar el MSV la organización lograra permanecer dentro del mercado adaptándose y transformándose a través del tiempo de acuerdo a los cambios del entorno. 


\section{CONCLUSIONES}

El Modelo del Sistema Viable implementado como herramienta cibernética permite el desarrollo de un diseño diagnóstico de organizaciones. Permitirá crear estrategias completas estructurando la organización basada en los objetivos que permitan establecer las formas de gestión adecuadas para estos. Con ello, permitirá dar ventaja en la formalización para la obtención del diagnóstico rápido del problema. Mediante cinco funciones permitirá generar diagnósticos y conceptualización de mejoras con la implementación administrando eficientemente los recursos para una presentación de resultados satisfaciendo las necesidades y objetivos, planteados por la coordinación que establece la realización de estos bajo la supervisión del control que asegura la eficiencia de la operación; la política que diseccionando los propósitos a evaluar dentro de la compañía para el alcance a través de la inteligencia con la planificación de un futuro viable.

Dentro del MSV es importante la opinión y cooperación de los colaboradores ya que intervienen directamente en los procesos, afectando el desarrollo y planeación futura de la compañía impactando la variedad de procesos que con la ayuda de estos enriquecen la información y desempeño de las funciones establecidas. Por ello, la inclusión de constantes capacitaciones permite adquirir conocimientos respecto a técnicas y recursos que forjan sentido de pertenencia mayor dentro de la organización para asumir el rol y actividad como principio fundamental para el correcto funcionamiento de la compañía basado en el constante crecimiento y mejora. 


\section{REFERENCIAS BIBLIOGRÁFICAS}

\section{Referencias}

Andes, U. p. (s.f.). Issuu. Obtenido de https://issuu.com/christianmb/docs/metodologia_de_sistemas-libro/95

Beer, S. (1985). Diagnosing the System for Organizations, John Wiley \& Sons, Chichester.

Beer, S. (1979). The Heart of enterprise. Jhon Wiley \& sons.

Espejo, R., \& Reyes, A. (2016). Sistemas organizacionales. El manejo de la complejidad con el modelo de sistema. Universidad de los Andes; Universidad de Ibagué.

Espinosa, A., \& Walker, J. (2011). A complexity approach to sustainability: Theory and application. 1 Ed. World Scientific.

Puche Regaliza, J. C., Pérez Ríos, J. M., \& Sánchez Mayoral, P. (2005). Proyectos Software desde una perspectiva cibernética. Obtenido de IX Congreso de Ingeniería de Organización:

http://www.adingor.es/congresos/web/uploads/cio/cio2005/prod_gest_operaciones/128.pd $\mathrm{f}$

Peón Escalante, I. E. (2015). Transformación integral de organizaciones complejas. Sociedad Cooperativa de Producción Taller.

Espinosa, A., \& Walker, J. (2017). A complexity approach to sustainability: Theory and application . Obtenido de World Scientific. : https://www.worldscientific.com/worldscibooks/10.1142/p699

Francis, H., Francis, M., Taylor, M., \& Kinloch, P. (2009). Supporting crime detection and operational planning with soft systems methodology and viable systems model. Obtenido de Wiley Online Library: https://onlinelibrary.wiley.com/doi/abs/10.1002/sres.943

perez Gomez, G. J. (2020). Diseño del Modelo Lista de verificacion. Bogotá D.C.: Universidad Autónoma de Colombia.

El Modelo de Sistema Viable. (s.f.). Obtenido de Quadernsdigitals: http://www.quadernsdigitals.net/datos_web/biblioteca/1_223/enLinea/21.htm 


\section{FIGURAS}

- Figura 1. Gerencia de complejidad (Espejo, R. 2003, Pérez, G. 2020)

- Figura 2. Gerencia de complejidad (Espejo, R. 2003, Pérez, G. 2020)

- Figura 3. Mecanismo de Cohesión. Canal de Comandos. (Espejo, R. 2003, Pérez, G.

2020)

- Figura 4. Mecanismo de Cohesión. Canal de Coordinación. (Espejo, R. 2003, Pérez, G. 2020)

- Figura 5. Mecanismo de Cohesión. Monitoreo de Actividades primarias. (Espejo, R.

2003, Pérez, G. 2020)

- Figura 6. Mecanismo de Cohesión. Completo. (Espejo, R. 2003, Pérez, G. 2020)

- Figura 7. Mecanismo de Adaptación. Completo. (Espejo, R. 2003, Pérez, G. 2020)

- Figura 8. El modelo del Sistema Viable al completo. (Beer, S. 1985)

\section{TABLAS}

Tabla 1: Criterios de calificación (Pérez, G. 2020) 